\title{
Cognitive flexibility depends on white matter microstructure of the basal ganglia
}

\author{
M.R. van Schouwenburg a,b,c,*, A.M.H. Onnink ${ }^{a}$, N. ter Huurne ${ }^{\mathrm{a}, \mathrm{b}}$, C.C. Kan ${ }^{\mathrm{a}}$, M.P. Zwiers ${ }^{\text {b,f }}$, \\ M. Hoogman ${ }^{\mathrm{a}, \mathrm{d}}$, B. Franke ${ }^{\mathrm{a}, \mathrm{e}}$, J.K. Buitelaar ${ }^{\mathrm{f}}$, R. Cools ${ }^{\mathrm{a}, \mathrm{b}}$ \\ ${ }^{a}$ Radboud University Medical Centre, Donders Institute for Brain, Cognition and Behaviour, Department of Psychiatry, PO Box 9101, \\ 6500 HB Nijmegen, The Netherlands \\ ${ }^{\mathrm{b}}$ Radboud University Nijmegen, Donders Institute for Brain, Cognition and Behaviour, Centre for Cognitive Neuroimaging, PO Box 9104 , \\ 6500 HE Nijmegen, The Netherlands \\ ${ }^{\mathrm{c}}$ Department of Neurology, University of California, San Francisco, CA 94158, USA \\ ${ }^{\mathrm{d}}$ Max Planck Institute for Psycholinguistics, Department of Language and Genetics, PO Box 310, 6500 AH Nijmegen, The Netherlands \\ e Radboud University Medical Centre, Donders Institute for Brain, Cognition and Behaviour, Department of Human Genetics, PO Box 9101, \\ 6500 HB Nijmegen, The Netherlands \\ ${ }^{\mathrm{f}}$ Radboud University Medical Centre, Donders Institute for Brain, Cognition and Behaviour, Department of Cognitive Neuroscience, PO Box 9104,6500 HE \\ Nijmegen, The Netherlands
}

\section{A R T I C L E I N F O}

\section{Article history:}

Received 3 April 2013

Received in revised form 3 November 2013

Accepted 18 November 2013

Available online 28 November 2013

Keywords:

Basal ganglia

Cognitive control

DTI

Structural connectivity

Fronto-striatal circuits ADHD

\begin{abstract}
A B S T R A C T
Ample evidence shows that the basal ganglia play an important role in cognitive flexibility. However, traditionally, cognitive processes have most commonly been associated with the prefrontal cortex. Indeed, current theoretical models of basal ganglia function suggest the basal ganglia interact with the prefrontal cortex and thalamus, via anatomical fronto-striato-thalamic circuits, to implement cognitive flexibility. Here we aimed to assess this hypothesis in humans by associating individual differences in cognitive flexibility with white matter microstructure of the basal ganglia. To this end we employed an attention switching paradigm in adults with ADHD and controls, leading to a broad range in task performance. Attention switching performance could be predicted based on individual differences in white matter microstructure in/around the basal ganglia. Crucially, local white matter showing this association projected to regions in the prefrontal cortex and thalamus. Our findings highlight the crucial role of the basal ganglia and the fronto-striato-thalamic circuit for cognitive flexibility.
\end{abstract}

(c) 2013 Elsevier Ltd. All rights reserved.

\section{Introduction}

Our constantly changing environment demands cognitive flexibility, i.e. the ability to switch attention away from previously relevant representations and towards newly relevant representations. Accumulating evidence suggests that such cognitive flexibility is supported by the basal ganglia. Thus, selective lesions of the basal ganglia in experimental animals impair set shifting and reversal learning (Crofts et al., 2001; Oberg \& Divac, 1975; Taghzouti, Louilot, Herman, Le Moal, \& Simon, 1985). In humans, functional magnetic resonance imaging (fMRI) studies have demonstrated BOLD signal increases in the basal ganglia during the performance of paradigms that require cognitive flexibility, such as task switching, reversal learning and set-shifting paradigms (Cools, Clark, Owen, \& Robbins, 2002; Cools, Clark, \& Robbins, 2004; Leber, Turk-Browne, \& Chun, 2008; Rogers, Andrews,

\footnotetext{
* Corresponding author at: University of California, San Francisco, Department of Neurology, San Francisco, California 94158, USA.

E-mail address: martine.vanschouwenburg@ucsf.edu (M.R. van Schouwenburg).
}

Grasby, Brooks, \& Robbins, 2000). Evidence that the basal ganglia are not just activated, but in fact necessary for cognitive flexibility in humans comes from studies with Parkinson's disease and stroke patients with focal basal ganglia lesions, who exhibit significant set switching deficits (Cools, Van den Bercken, Horstink, Van Spaendonck, \& Berger, 1984; Cools, Barker, Sahakian, \& Robbins, 2001; Cools, Ivry, \& D’Esposito, 2006; Downes et al., 1989; Owen et al., 1992).

The above described studies convincingly show a role for the basal ganglia in cognitive flexibility. This might seem surprising in the context of classic models of cognitive control that emphasize a particularly important role for the prefrontal cortex (Miller \& Cohen, 2001; Milner, 1963; Owen, Roberts, Hodges, \& Robbins, 1993; Rogers, 1998). In fact there is a long history of research on the similarities between the functional consequences of frontal and basal ganglia lesions (Divac, 1972). Based on this classic work, the functions of the basal ganglia have been hypothesized to be determined by its cortical and possibly by its thalamic input (Divac, 1972). Anatomical evidence for the existence of strong white matter connections between these regions in fronto-striatothalamic circuits (Alexander, DeLong, \& Strick, 1986; Draganski 
et al., 2008) strengthened this hypothesis, which is also in line with more recent theoretical work, suggesting more explicitly that cognitive flexibility depends on interactions between the basal ganglia, the prefrontal cortex and the thalamus (Frank, Loughry, \& O'Reilly, 2001; Hazy, Frank, \& O'Reilly, 2007). However, despite this strong anatomical and theoretical basis, there is no direct evidence for the importance of structural connectivity of the basal ganglia for cognitive flexibility. Here we establish this link between cognitive flexibility and individual differences in white matter microstructure of the human basal ganglia by employing diffusion tensor imaging (DTI).

An attention switching paradigm was used to assess cognitive flexibility. This paradigm was previously shown to reliably recruit the basal ganglia during a switch of attention (van Schouwenburg, den Ouden, \& Cools, 2010; van Schouwenburg et al., 2013). White matter microstructure of the basal ganglia was indexed by fractional anisotropy (FA), measured with DTI. We and others have used this approach previously to link cognitive measures to white matter microstructure (Boorman, O'Shea, Sebastian, Rushworth, \& Johansen-Berg, 2007; Forstmann et al., 2008; Neubert, Mars, Buch, Olivier, \& Rushworth, 2010; Tuch et al., 2005). For example, we have shown, using the same attention switching paradigm, that BOLD signal in the basal ganglia depends on individual differences in FA values in the basal ganglia (van Schouwenburg et al., 2013). We now aim to extend this prior work to individual differences in performance on the task.

In our previous study, which included only healthy subjects, the narrow distribution of task performance prevented us from assessing this relationship between white matter microstructure and performance. Here, we anticipated that the inclusion of subjects diagnosed with attention deficit hyperactivity disorder (ADHD) would lead to a broader range of task performance. ADHD has previously been associated with performance deficits on setshifting (Boonstra, Kooij, Oosterlaan, Sergeant, \& Buitelaar, 2010; Boonstra, Oosterlaan, Sergeant, \& Buitelaar, 2005) and task switching paradigms (King, Colla, Brass, Heuser, \& Von Cramon, 2007; McLean et al., 2004), suggesting that ADHD is accompanied by cognitive inflexibility.

\section{Methods}

\subsection{Subjects}

Nineteen healthy volunteers and 19 volunteers diagnosed with ADHD were recruited from an existing database (Dutch cohort of the International Multicenter persistent ADHD CollaboraTion (IMpACT) (Hoogman et al., 2011)). All participants were assessed using the Diagnostic Interview for Adult ADHD at the time of inclusion in the IMpACT study (Kooij \& Francken, 2007). This interview focuses on the 18 DSM-IV symptoms of ADHD and uses concrete and realistic examples to thoroughly investigate whether a symptom is currently present or was present in childhood. The Structured Clinical Interview for DSM-IV (SCID-I) was used for comorbidity assessment. Assessments were carried out by trained professionals (psychiatrists or psychologists).

All subjects gave written informed consent and were compensated for participation. The study was approved by the local ethics committee (committee for the protection of human subjects of the Arnhem/Nijmegen region; CMO protocol number 2009/260)

All 38 subjects performed the attention switching paradigm as described below. None of the subjects participated in any of our previous studies using the same paradigm (van Schouwenburg et al., 2010, 2013). Subjects were asked to complete the ADHD DSM-IV-TR Rating Scale (ADRS) at home and to bring it with them on the day of testing (Kooij et al., 2005). This self-report questionnaire was used to assess inattentive symptoms and hyperactivity symptoms in the last six months. ADRS data were missing from two subjects (one control subject and one ADHD patient). Structural MRI and diffusion tensor images were missing for five additional subjects (three control subjects and two ADHD patients). Accordingly, results are reported from 31 subjects. These 31 subjects included 15 control subjects ( 9 men), and 16 ADHD patients ( 7 men). None of the volunteers had (co-morbid) psychiatric or neurological disorders at the time of testing. There were no significant differences between the ADHD and control group with respect to
Table 1

Demographics of ADHD patients and healthy controls.

\begin{tabular}{|c|c|c|c|c|}
\hline & \multicolumn{2}{|c|}{ ADHD $(n=16)$} & \multicolumn{2}{|c|}{ Control $(n=15)$} \\
\hline & Mean & SEM & Mean & SEM \\
\hline Age & 32.5 & 1.7 & 31.6 & 1.4 \\
\hline $\mathbf{I Q}^{\mathbf{a}}$ & 11.6 & 0.6 & 11.5 & 0.7 \\
\hline Inattentive symptoms & 5.7 & 0.7 & 0.8 & 0.4 \\
\hline $\begin{array}{l}\text { Hyperactivity/Impulsivity } \\
\text { symptoms }\end{array}$ & 3.9 & 0.6 & 0.9 & 0.2 \\
\hline
\end{tabular}

a Scores represent the average of the standard scores for the block design and vocabulary assessments of the Wechsler Adult Intelligence Scale-III.

gender $\left(X^{2}=1.57, p=0.21\right)$, age $\left(t_{29}=-0.40, p=0.69\right)$, or IQ $\left(t_{29}=-0.03, p=0.97\right)$ (Table 1). Four of the ADHD patients were medication-naive and three had taken medication in the past, but were off medication at the time of the experiment. The remaining nine ADHD patients took regular medication, but withdrew from medication approximately $24 \mathrm{~h}$ prior to the experiment.

\subsection{Paradigm}

An attention switching paradigm was employed in which subjects switched attention when they detected a change in the stimulus exemplars of a non-selected category of face/scene stimuli (van Schouwenburg et al., 2010). Subjects were presented with a series of stimulus-pairs, each consisting of a superimposed face exemplar and scene exemplar (Fig. 1A). Subjects were instructed to select one of four exemplars by making a left (left index finger) or right (right index finger) response, depending on the location of the exemplar of their choice. This self chosen exemplar was then set as the correct stimulus and subjects were instructed to continue selecting that stimulus on subsequent trials. Stimulus-pairs were presented twice within each trial and subjects were instructed to select the same stimulus on both presentations within a trial. The specific pairing of the superimposed face and scene exemplars was opposite on the second presentation relative to the first presentation (e.g. if face 1 overlapped scene 1 on the first presentation, then face 1 overlapped scene 2 on the second presentation), enabling us to identify which stimulus exemplar was selected by the subject (Fig. 1). Feedback was presented after each trial, and was positive only if the subject selected the correct stimulus twice within the trial. If subjects selected the pattern that did not contain the correct exemplar or did not respond within a personalized cut-off time, then negative feedback was presented.

After a variable number of correct trials, exemplars of the ignored category were replaced with novel exemplars. Subjects were instructed prior to the experiment to switch attention to this other category, and to choose one of the two novel exemplars, as soon as they detected a change. Trials on which novel exemplars were introduced, and on which subjects detected the change and switched to one of the novel exemplars where classified as novel switch trials (Fig. 1C). On some trials subjects failed to detect the novel exemplars and continued to respond to the previously correct exemplar (novel non-switch trials) (Fig. 1D). In this case negative feedback was presented, usually leading subjects to switch on the subsequent trial. Trials on which no novel stimuli were introduced were defined as repeat trials (Fig. 1B). For more details about the paradigm we refer to our previous study (van Schouwenburg et al., 2010).

Subjects performed a practice block before the start of the main experiment, consisting of on average 140.2 trials $( \pm 4.5$ [SEM]). During the main experiment, subjects were presented with an average of 405.0 repeat trials $( \pm 11.5)$ (control: $401.0 \pm 14.6$, ADHD: $408.7 \pm 18.0$ ), and novel exemplars were introduced on 82 trials. The sequence of the faces and scenes presented was randomized across subjects but were matched between groups. The timing of the paradigm was slightly adjusted compared to our previous study. Time between presentation of the first and second stimulus was reduced to $500 \mathrm{~ms}$ (previously $1000 \mathrm{~ms}$ ) and feedback was given immediately after the second response (previously jittered between 0 and $4500 \mathrm{~ms}$ to allow for desynchronization of trials necessary for fMR analyses). These adjustments reduced the duration of the experiment by approximately $15 \mathrm{~min}$ (total current duration: 25-30 min).

The paradigm was programmed using Presentation software (Neurobehavioural systems, Albany, USA).

\subsection{MRI data acquisition}

Whole-brain imaging was performed with a $1.5 \mathrm{~T}$ MR scanner (Magnetom Avanto, Siemens Medical Systems, Erlangen, Germany) at the time of inclusion in the IMpACT study (0-3.5 years prior to the current experiment) (Hoogman et al, 2011). A high-resolution T1-weighted MP-RAGE anatomical scan was obtained from each subject (176 sagittal slices, repetition time $=2730 \mathrm{~ms}$, echo time $=2.95 \mathrm{~ms}$, voxel size $=1.0 \times 1.0 \times 1.0 \mathrm{~mm}$, field of view $=256 \mathrm{~mm}$ ). In addition, diffusion tensor 


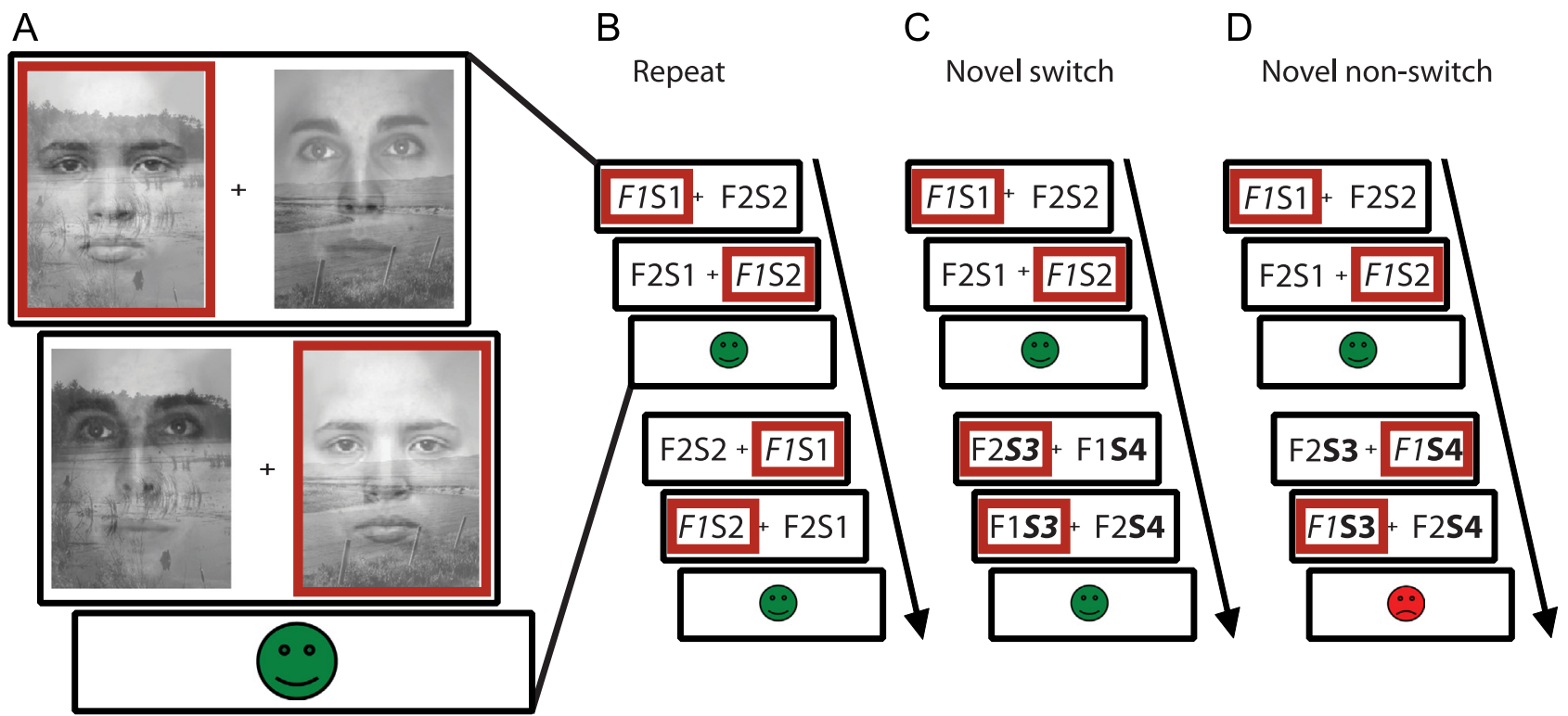

Fig. 1. The attention-switching paradigm used in this study required subjects to select one stimulus exemplar (left versus right) within one dimension (faces versus scenes) on every trial. (A) Each trial consisted of two consecutive responses followed by feedback. Red boxes indicate a possible response sequence. (B)-(D) show two consecutive trials with responses defining the three different trial types. For clarification, the stimuli are displayed schematically (F1, face 1; S1, scene1; F2, face 2; S2, scene 2). (B) In this example, the subject is attending to F1 on the first trial (attended stimuli are displayed in italic). On the next trial, no novel stimuli are introduced and the subject continues to attend to F1. The second trial is thus defined as a repeat trial. (C) On a novel switch trial, novel stimuli of the unattended dimension, in this case scenes, are introduced (S3 and S4). The subject detects this change and switches attention to one of two novel stimuli (here S3). (D) Alternatively the subject can fail to detect the novel stimuli and continue to respond to the previously relevant stimulus exemplar, in this case F1. The subject will then receive negative feedback and the second trial is defined as a novel non-switch trial. (For interpretation of the references to color in this figure legend, the reader is referred to the web version of this article.)

images were acquired using a twice-refocused spin-echo echo-planar imaging sequence. Eighteen subjects ( 8 controls, 10 ADHD) were scanned with the following protocol: 56 slices interleaved acquisition mode, repetition time $=6700 \mathrm{~ms}$, echo time $=85 \mathrm{~ms}$, voxel size $=2.5 \times 2.5 \times 2.5 \mathrm{~mm}$, field of view $=220 \mathrm{~mm}$. Four images without diffusion weighting $(b=0)$, and 30 images with diffusion weighting $\left(b=1000 \mathrm{~s} / \mathrm{mm}^{2}\right)$ applied along non-colinear directions were acquired. The remaining 13 subjects ( 7 controls, 5 ADHD) were scanned with an adapted second diffusion weighted acquisition protocol, which was implemented to reduce motion artifacts during scanning (64 slices interleaved acquisition mode, repetition time $=10200 \mathrm{~ms}$, echo time $=95 \mathrm{~ms}$, voxel size $=2.5 \times 2.5 \times 2.5 \mathrm{~mm}$, field of view $=320 \mathrm{~mm})$. Four images without diffusion weighting $(b=0)$, and 30 images with diffusion weighting $\left(b=900 \mathrm{~s} / \mathrm{mm}^{2}\right)$ applied along non-colinear directions were acquired. We corrected for possible variance introduced by using different protocols where appropriate by including DTI protocol as a covariate.

\subsection{Diffusion tensor imaging (DTI) analysis}

DTI images were realigned and eddy-current corrected by residual error minimization of the diffusion tensor model (Andersson \& Skare, 2002). Susceptibility induced echo-planar imaging distortions were corrected by warping the images to the distortion-free T1 reference image (Studholme, Constable, \& Duncan, 2000) using an in-house developed implementation (Visser, Qin, \& Zwiers, 2010) in SPM8 software (Statistical Parametric Mapping; Wellcome Trust Centre for Cognitive Neuroimaging, London, UK.

Diffusion tensors were then robustly estimated using an artefact-insensitive compute algorithm (Zwiers, 2010). Fractional anisotropy (FA) values were computed from the diffusion tensor eigenvalues. FA maps were normalized to the T1 ICBM-template (MNI space) using the unified segmentation parameters of the anatomical T1 image (Ashburner \& Friston, 2005), and spatially smoothed using a Gaussian kernel of $8 \mathrm{~mm}$ full width at half maximum.

To investigate the question whether white matter microstructure is associated with cognitive flexibility, we assessed the switch likelihood for each subject as a measure of cognitive flexibility. Switch likelihood was calculated as the percentage of immediate switches in response to a novel stimulus. We submitted the normalized FA maps to a second level one-sample $t$-test, with switch likelihood as a covariate of interest and DTI scanning protocol as a covariate of non-interest. In an additional analysis we included four more covariates of non-interest: sex, age, intracranial volume and diagnosis (ADHD vs. control) to ensure our results were not driven by any of these factors.

FA results were masked by a whole brain mask and a threshold mask of FA $>0.2$. Previously we had shown that individual differences in (drug effects on) basal ganglia BOLD signal during attention switching correlated with FA values in the basal ganglia (van Schouwenburg et al., 2013). More specifically, a whole-brain analysis revealed a significant FA cluster selectively in the basal ganglia. This prior work provided the basis for our current hypothesis that FA values in this region might also correlate with individual differences in attention switching in behavioural terms. Accordingly, we defined our FA volume of interest (VOI) based on this previous study (van Schouwenburg et al., 2013). Specifically, we defined our FA VOI as a 4-mm sphere around the peak coordinates [18 60 ] found previously in the right basal ganglia and mirrored this to obtain an FA VOI in the left basal ganglia $[-1860]$. These two were then combined into one FA VOI, containing a cluster in the left and right basal ganglia (we had no a priori hypothesis on hemisphere selective effects). Definition of VOI's and VOI data extraction were done using MarsBaR (Brett, Anton, Valabregue, \& Poline, 2002). Extracted data were further analysed using Pearson's correlation in SPSS (SPSS Inc. 15.0.0 for Windows).

Correlations between FA values and switch likelihood were assessed at the voxel-level, corrected for multiple comparisons across our VOI in the basal ganglia $\left(p_{\mathrm{svc}}<0.05\right)$. In addition, FA values were extracted from our VOI and averaged across voxels to assess and to plot the correlation between FA value and switch likelihood. Note that the VOI was defined a priori based on an independent study and therefore is not biased towards finding a significant correlation between switch likelihood and the extracted FA data.

Additional exploratory analyses were performed across the whole brain ( $\left.p_{\text {uncorr }}<0.001\right)$ for completeness.

\subsection{Fibre tracking}

The FA region showing a significant correlation with switch likelihood was used for probabilistic diffusion tractography to identify white matter tracts connecting with this location. More specifically, we defined a VOI as a 4-mm sphere around the peak voxels of the correlations (MNI coordinates [-18 2 0] and [20 4 2]). For each subject this VOI was brought back into native space, using the inverse of the computed normalization parameters. FMRIB's Diffusion Toolbox (part of FMRIB's Software Library (FSL)) was used to build up distributions on diffusion parameters at each voxel, allowing for crossing fibres (using 'bedpostx') (Behrens, Berg, Jbabdi, Rushworth, \& Woolrich, 2007), and subsequent probabilistic tracking from the VOI to all other voxels in the brain (using 'probtrackx' with standard settings). To eliminate spurious connections, tractography in individual subjects was thresholded to include only voxels through which at least 50 samples had passed (out of 5000). These individual tracts were then binarized and summed across subjects to produce group probability maps. In these maps, each voxel value represents the number of subjects in whom the pathway passes through that voxel. Results were thresholded to display only those paths that were present in at least $25 \%$ of the subjects ( 8 out of 31 ). 


\subsection{Behavioural analysis}

To assess between-group differences in terms of switch likelihood an independent sample $t$-test was performed. In addition, we assessed correlations between switch likelihood and ADRS scores using Pearson's correlation analyses in SPSS. For this analysis the five subjects who did complete the ADRS, but for whom MR data were missing were included.

Furthermore, median reaction times (RT) were calculated for our three trial types of interest (novel switch, novel non-switch and repeat). Excluded from these reaction time analyses were the first trial of each block, all trials on which subjects received negative feedback and trials following negative feedback. For each subject, we calculated the median reaction time, rather than mean reaction time, to minimize the influence of outliers. Four subjects with extreme switch likelihoods were excluded for this RT analysis due to a small number of (i.e. less than 10) novel switch or novel non-switch trials ( 3 controls and 1 ADHD). RT data were analysed with repeated-measures ANOVA with trial type (novel switch, novel non-switch, repeat) as a within-subject factor and group (ADHD, control) as a between-subject factor in SPSS

Results are reported as the mean \pm SEM across subjects. The statistical threshold was set at $p<0.05$ (two-tailed)

\section{Results}

\subsection{Brain-behaviour correlation}

To assess the hypothesis that attention switching performance is related to white matter in/around the basal ganglia, we correlated individual differences in switch likelihood with whole-brain FA values (corrected for DTI protocol). Such a correlational approach was possible because of the broad range in switch likelihood, which was achieved by the inclusion of ADHD patients and older healthy subjects, compared to our previous study. Consistent with our prediction we found a significant correlation with FA values in the basal ganglia (cluster 1 : $[-1820], t=3.65, p_{\mathrm{svc}}=0.009$, cluster 2 : [20 42 ], $t=3.34, p_{\mathrm{svc}}=0.018$ ) across subjects (controls and ADHD) (Fig. 2). Clusters were centred around the left and right pallidum. Whole-brain results at $p_{\text {uncorrected }}<0.001$ are reported in Table 2 for completeness. In an additional analysis we included diagnosis, sex, age and intracranial volume as regressors. This analysis yielded similar results (cluster 1 : $[-1820], t=2.93, p_{\mathrm{svc}}=0.053$, cluster 2 : [20 4 2], $t=3.09, p_{\mathrm{svc}}=0.038$ ). This suggest that our results are not confounded by any of these factors.

The correlation was positive, such that high local FA values were associated with high switch likelihood. Averaged data extracted from the basal ganglia VOI confirmed these findings by showing a significant between-subjects correlation with switch likelihood $\left(r_{28}=0.475, p=0.008\right.$, corrected for DTI protocol) (Fig. 2).

\subsection{Probabilistic diffusion tractography}

To identify the white matter tracts connecting with the region in which the switch likelihood-FA association was found, we used the cluster found in the basal ganglia as a seed region for probabilistic diffusion tractography (Behrens et al., 2007). White matter tracts ran from the basal ganglia to the frontal cortex and from the basal ganglia to the thalamus, extending into the midbrain (Fig. 3), replicating our prior findings (van Schouwenburg et al., 2013).

\subsection{Behaviour}

Switch likelihood was not significantly different between the control subjects and subjects diagnosed with ADHD $\left(t_{29}=0.27\right.$, $p=0.79$ ) (control, $49.1 \pm 6.3 \%, \mathrm{ADHD}, 47.0 \pm 4.9 \%$ ). In addition, RT analyses revealed that there was also no significant interaction between trial type and group (trial type $\mathrm{x}$ group interaction: $F_{1,26}=1.18, \quad p=0.32$ )(control, switch: $1475 \pm 191 \mathrm{~ms}$, nonswitch: $948 \pm 124$ ms, repeat: $867 \pm 94 \mathrm{~ms}$ ) (ADHD, switch: $1504 \pm 134 \mathrm{~ms}$, nonswitch: $773 \pm 34 \mathrm{~ms}$, repeat: $780 \pm 38 \mathrm{~ms}$ ). These results indicate that ADHD patients were not improved or impaired relative to controls in terms of attention switching. However, we did find a significant correlation between switch likelihood and self-reported inattentive symptoms in ADHD subjects $\left(r_{16}=-0.52, p=0.027\right)$ (Fig. 4). More inattentive symptoms were associated with greater attention switching deficits in ADHD patients. In contrast, there was no such correlation with hyperactivity symptoms $\left(r_{16}=-0.08\right.$, $p=0.74)$. Furthermore, no correlations were found in the control group (inattentive symptoms: $r_{16}=0.13, p=0.62$, hyperactive symptoms: $r_{16}=0.12, p=0.65$ ), which is not surprising because there was hardly any variability in ADRS scores in this control group (Fig. 4).

Table 2

Clusters showing a correlation between switch likelihood and FA values at $p<0.001$ uncorrected, with a contiguous voxel cluster threshold $k>5$.

\begin{tabular}{|c|c|c|c|c|c|}
\hline \multirow[t]{2}{*}{ Region } & \multirow[t]{2}{*}{ Clustersize } & \multicolumn{3}{|c|}{ Local maximum } & \multirow{2}{*}{$\begin{array}{l}\text { Cluster } \\
\text { statistics } \\
t \text {-Value }\end{array}$} \\
\hline & & $x$ & $y$ & $z$ & \\
\hline Pallidum & 23 & -16 & 0 & 0 & 4.23 \\
\hline Cerebellum & 38 & -8 & -44 & -24 & 4.12 \\
\hline Amygdala & 14 & -24 & -2 & -14 & 3.92 \\
\hline Orbitofrontal cortex & 7 & 24 & 30 & -8 & 3.85 \\
\hline
\end{tabular}

A

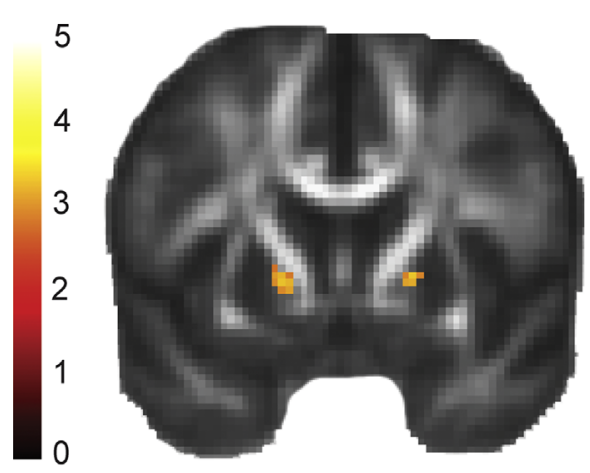

B

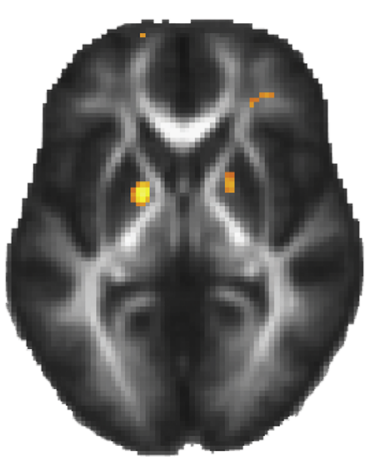

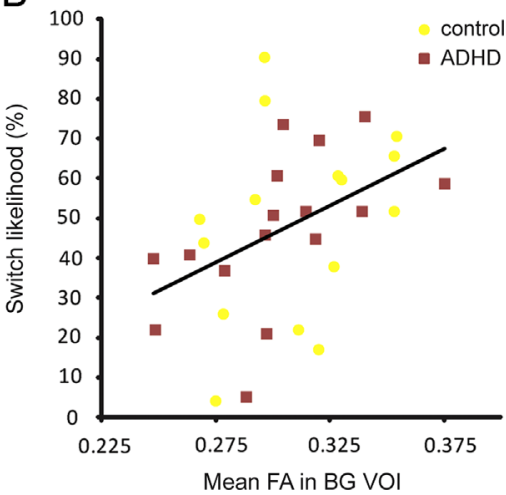

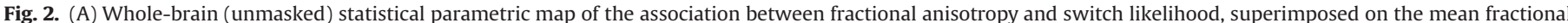

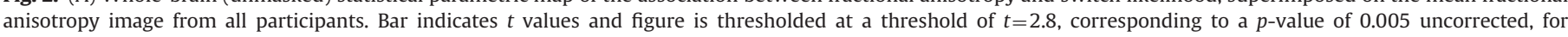

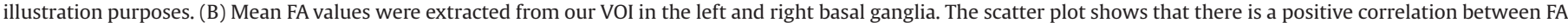
values in the basal ganglia and performance on the attention switching task across all participants. 

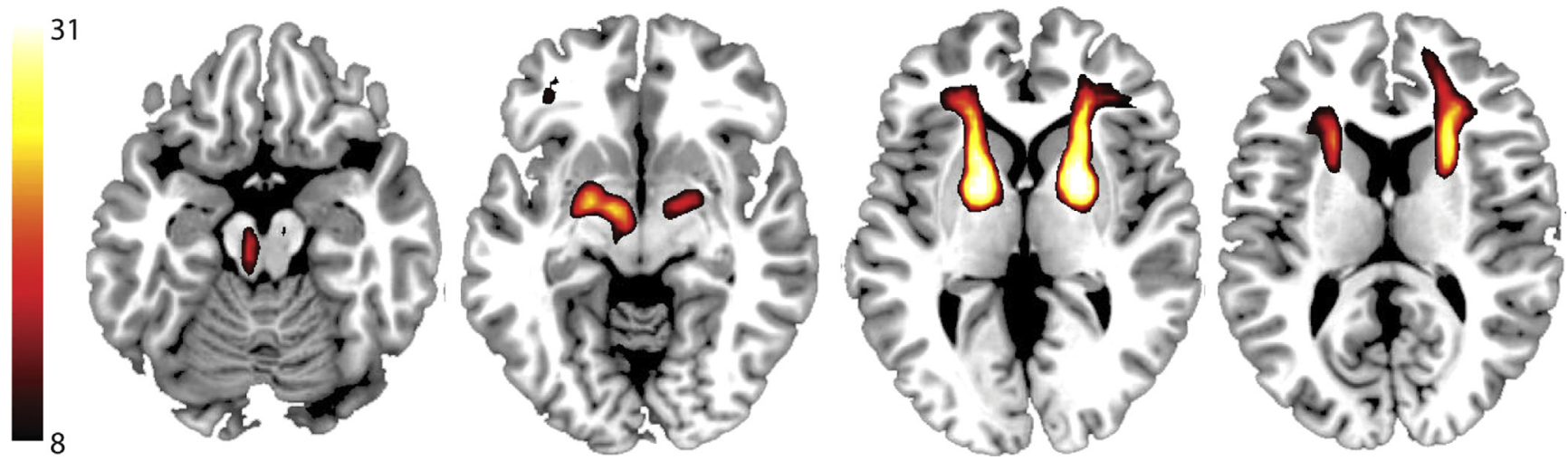

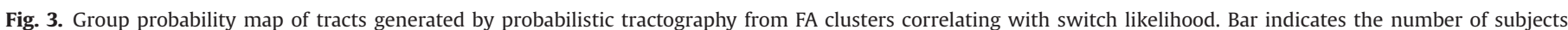
containing the path and the map is thresholded such that only tracts that were found in at least $25 \%$ of the subjects ( 8 out of 31 ) are included.

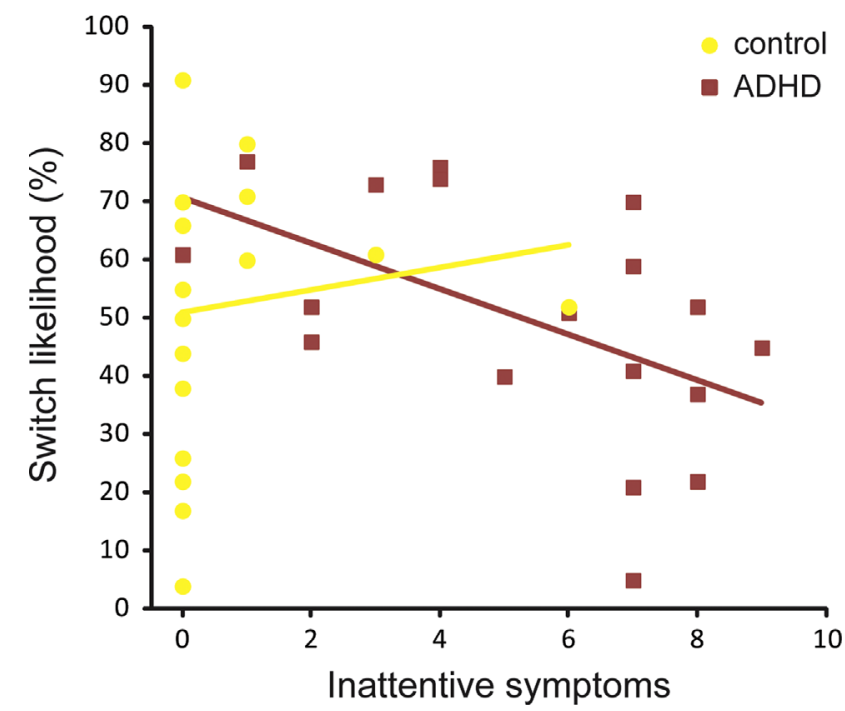

Fig. 4. Correlation between inattentive symptoms as scored on the ADRS and switch likelihood. A greater number of inattentive symptoms was associated with lower switch likelihood, thus impaired attention switching, but only in ADHD patients.

\section{Discussion}

Cognitive flexibility has traditionally been associated with the prefrontal cortex (Milner, 1963; Owen et al., 1993; Rougier, Noelle, Braver, Cohen, \& O'Reilly, 2005). However, evidence indicates that it does not act in isolation, but interacts with the subcortical basal ganglia to control attention switching (e.g. Hazy et al., 2007; van Schouwenburg et al., 2010). We demonstrate that attention switching performance can be predicted based on individual differences in white matter microstructure in/around the basal ganglia. Our data extend previous studies implicating the basal ganglia in attention switching (Cools et al., 2002, 2004, 2006; Crofts et al., 2001; Leber et al., 2008; Rogers et al., 2000) by linking it to white matter microstructure of the intact human basal ganglia.

Our approach resembles the approach that was used previously to link anatomical microstructure with individual differences in behaviour (Forstmann et al., 2008; Tuch et al., 2005). More specifically, we calculated fractional anisotropy values based on diffusion tensor images as an index of local white matter strength. Previous studies revealed that fractional anisotropy relies on several microstructural properties of white matter tissue, such as the level of axon myelination, intact axonal membranes, fibre density and fibre diameter (Beaulieu, 2002). This suggests that performance on our attention switching task might be associated with the level of neuronal communication within the basal ganglia, and/or between the basal ganglia and other brain regions.

The prefrontal cortex might be one such region. The basal ganglia and prefrontal cortex are strongly anatomically connected via fronto-striato-thalamic circuits (Alexander et al., 1986) and have been suggested to interact during attention switching (Frank, 2011; Hazy et al., 2007). Indeed, we have previously demonstrated that the basal ganglia act as a gate to selectively guide prefrontal representations during a switch in attention (van Schouwenburg et al., 2010). In line with the proposed role for fronto-striatal interaction in attention switching, probabilistic tractography from the basal ganglia region showing the FA-switch likelihood correlation, revealed a fronto-striato-thalamic network. This same network was found in our previous study (van Schouwenburg et al., 2013). In that study we showed that (drug effects on) switchrelated BOLD signal in the basal ganglia correlated with FA values in the exact same white matter region as found here. In addition, drug effects on functional (switch-related) fronto-striatal connectivity were correlated with the same FA values. However, the limited variance in task performance in that study prevented us from demonstrating correlations between task performance and white matter strength. In the present study we have increased the variance in task performance by including older healthy participants and ADHD patients and confirm the hypothesis that white matter microstructure of the basal ganglia, likely representing the degree to which it is connected to other brain regions, is necessary for optimal attention switching performance.

We did not find any differences in task performance between ADHD patients and controls, although there was a significant correlation between switch likelihood and inattentive symptoms within the ADHD group. More specifically, ADHD patients with more inattentive symptoms performed more poorly on our attention switching paradigm. This correlation was not found for the hyperactive symptoms. Hence, in line with previous studies, inattention was associated with impaired cognitive flexibility (Boonstra et al., 2010, 2005; King et al., 2007; McLean et al., 2004). In this context, it is interesting that several studies have reported decreased frontostriatal connectivity in ADHD patients (Cubillo, Halari, Smith, Taylor, \& Rubia, 2011; Cubillo \& Rubia, 2010; Konrad \& Eickhoff, 2010). Thus deficient structural and functional connectivity between the prefrontal cortex and basal ganglia in ADHD might underlie the observed deficits in cognitive flexibility (Cubillo et al., 2011; Cubillo \& Rubia, 2010; Konrad \& Eickhoff, 2010). In the current study we found no significant difference between the control group and the ADHD group in FA value within our basal ganglia VOI $(p=0.65)$, allowing us to assess the correlation between switch likelihood and basal ganglia FA values across all participants. It might be noted that ADHD symptom scores were rather low in our sample. Had we 
included more severe patients we might have found overall group differences.

A correlation between task performance and inattentive symptoms was not found in the control subjects. The failure to find such a correlation in the control group might reflect the fact that these subjects on average scored very low on the inattentive symptoms scale. In fact, most healthy subjects had a score of zero. It should be noted that this questionnaire was developed for clinical purposes and might be less appropriate for use in healthy individuals. Thus, questions are formulated to assess whether inattentive symptoms form a problem in daily life. Moreover, subjects were informed that they participated in an ADHD study, which might have biased the control subjects to report low inattentive symptoms. In a follow-up study we could use an alternative questionnaire to score distractibility and inattentive symptoms to assess whether there is a correlation between switch likelihood and inattentive symptoms in healthy controls as well.

The present study investigated cognitive flexibility with a task that required a switch in attention in response to novel stimuli. Such switching depends on multiple subcomponent processes, including novelty detection, inhibition of a previous stimulusresponse association, and selection of a novel stimulus. We did not aim to isolate the specific subcomponent process that was associated with basal ganglia white matter microstructure, but rather we aimed to assess cognitive flexibility performance using an ecologically valid model of attention switching.

In conclusion, we have shown that attention switching depends on white matter microstructure in/around the basal ganglia. This finding supports the idea that the basal ganglia, and their interaction with other brain regions, are involved in attention switching. In addition, we demonstrated that inattentive symptoms in ADHD were associated with performance deficits on the attention switching task.

\section{References}

Alexander, G. E., DeLong, M. R., \& Strick, P. L. (1986). Parallel organization of functionally segregated circuits linking basal ganglia and cortex. Annual Review of Neuroscience, 9, 357-381.

Andersson, J. L., \& Skare, S. (2002). A model-based method for retrospective correction of geometric distortions in diffusion-weighted EPI. NeuroImage, 16(1), 177-199.

Ashburner, J., \& Friston, K. J. (2005). Unified segmentation. NeuroImage, 26(3), 839-851.

Beaulieu, C. (2002). The basis of anisotropic water diffusion in the nervous system a technical review. NMR in Biomedicine, 15(7-8), 435-455.

Behrens, T. E., Berg, H. J., Jbabdi, S., Rushworth, M. F., \& Woolrich, M. W. (2007). Probabilistic diffusion tractography with multiple fibre orientations: What can we gain? Neurolmage, 34(1), 144-155.

Boonstra, A. M., Kooij, J. J. S., Oosterlaan, J., Sergeant, J. A., \& Buitelaar, J. K. (2010). To act or not to act, that's the problem: Primarily inhibition difficulties in adult ADHD. Neuropsychology, 24(2), 209-221.

Boonstra, A. M., Oosterlaan, J., Sergeant, J. A., \& Buitelaar, J. K. (2005). Executive functioning in adult ADHD: A meta-analytic review. Psychological Medicine, 35 (8), 1097-1108.

Boorman, E. D., O'Shea, J., Sebastian, C., Rushworth, M. F. S., \& Johansen-Berg, H. (2007). Individual differences in white-matter microstructure reflect variation in functional connectivity during choice. Current Biology, 17(16), 1426-1431.

Brett, M., Anton, J.L., Valabregue, R., \& Poline, J.B. (2002). Region of interest analysis using an SPM toolbox [abstract]. In: Presented at the eighth international conference on functional mapping of the human brain, June 2-6, 2002, Sendai, Japan.

Cools, A. R., Van den Bercken, J. H., Horstink, M. W., Van Spaendonck, K. P., \& Berger, H. J. (1984). Cognitive and motor shifting aptitude disorder in Parkinson's disease. Journal of Neurology, Neurosurgery \& Psychiatry, 47(5), 443-453.

Cools, R., Barker, R. A., Sahakian, B. J., \& Robbins, T. W. (2001). Enhanced or impaired cognitive function in Parkinson's disease as a function of dopaminergic medication and task demands. Cerebral Cortex, 11(12), 1136-1143.

Cools, R., Clark, L., Owen, A. M., \& Robbins, T. W. (2002). Defining the neural mechanisms of probabilistic reversal learning using event-related functional magnetic resonance imaging. Journal of Neuroscience, 22(11), 4563-4567.
Cools, R., Clark, L., \& Robbins, T. W. (2004). Differential responses in human striatum and prefrontal cortex to changes in object and rule relevance. Journal of Neuroscience, 24(5), 1129-1135.

Cools, R., Ivry, R. B., \& D'Esposito, M. (2006). The human striatum is necessary for responding to changes in stimulus relevance. Journal of Cognitive Neuroscience, 18(12), 1973-1983.

Crofts, H. S., Dalley, J. W., Collins, P., Van Denderen, J. C. M., Everitt, B. J., Robbins, T. W. et al. (2001). Differential effects of 6-OHDA lesions of the frontal cortex and caudate nucleus on the ability to acquire an attentional set. Cerebral Cortex, 11(11), 1015-1026.

Cubillo, A., Halari, R., Smith, A., Taylor, E., \& Rubia, K. (2011). A review of frontostriatal and fronto-cortical brain abnormalities in children and adults with Attention Deficit Hyperactivity Disorder (ADHD) and new evidence for dysfunction in adults with ADHD during motivation and attention. Cortex, 48(2).

Cubillo, A., \& Rubia, K. (2010). Structural and functional brain imaging in adult attention-deficit/hyperactivity disorder. Expert Review of Neurotherapeutics, 10 (4), 603-620.

Divac, I. (1972). Delayed alternation in cats with lesions of the prefrontal cortex and the caudate nucleus. Physiology \& Behavior, 8(3), 519-522.

Downes, J., Roberts, A., Sahakian, B., Evenden, J., Morris, R., \& Robbins, T. (1989) Impaired extra-dimensional shift performance in medicated and unmedicated Parkinson's disease: Evidence for a specific attentional dysfunction. Neuropsychologia, 27(11-12), 1329-1343.

Draganski, B., Kherif, F., Klöppel, S., Cook, P. A., Alexander, D. C., Parker, G. J. M., et al. (2008). Evidence for segregated and integrative connectivity patterns in the human Basal Ganglia. Journal of Neuroscience, 28(28), 7143-7152.

Forstmann, B. U., Jahfari, S., Scholte, H. S., Wolfensteller, U., van den Wildenberg, W. P., \& Ridderinkhof, K. R. (2008). Function and structure of the right inferior frontal cortex predict individual differences in response inhibition: A modelbased approach. Journal of Neuroscience, 28(39), 9790-9796.

Frank, M. J. (2011). Computational models of motivated action selection in corticostriatal circuits. Current Opinion in Neurobiology, 21(3), 1-6.

Frank, M. J., Loughry, B., \& O’Reilly, R. C. (2001). Interactions between frontal cortex and basal ganglia in working memory: A computational model. Cognitive, Affective, E' Behavioral Neuroscience, 1(2), 137-160.

Hazy, T. E., Frank, M. J., \& O’Reilly, R. C. (2007). Towards an executive without a homunculus: Computational models of the prefrontal cortex/basal ganglia system. Philosophical Transactions of the Royal Society of London, Series B, 362 (1485), 1601-1613.

Hoogman, M., Aarts, E., Zwiers, M., Slaats-Willemse, D., Naber, M., Onnink, M., et al (2011). Nitric oxide synthase genotype modulation of impulsivity and ventra striatal activity in adult ADHD patients and healthy comparison subjects. American Journal of Psychiatry, 168(10), 1099-1106.

King, J. A., Colla, M., Brass, M., Heuser, I., \& Von Cramon, D. (2007). Inefficient cognitive control in adult ADHD: Evidence from trial-by-trial Stroop test and cued task switching performance. Behavioral and Brain Functions, 3, 42.

Konrad, K., \& Eickhoff, S. B. (2010). Is the ADHD brain wired differently? A review on structural and functional connectivity in attention deficit hyperactivity disorder. Human Brain Mapping, 31(6), 904-916.

Kooij, J. J. S., Buitelaar, J. K., Van den Oord, E. J., Furer, J. W., Rijnders, C. A. T., \& Hodiamont, P. P. G. (2005). Internal and external validity of attention-deficit hyperactivity disorder in a population-based sample of adults. Psychological Medicine, 35(6), 817-827.

Kooij, J.J.S., \& Francken, M.H. (2007). Diagnostisch interview voor ADHD (DIVA) bij volwassenen.

Leber, A. B., Turk-Browne, N. B., \& Chun, M. M. (2008). Neural predictors of momentto-moment fluctuations in cognitive flexibility. Proceedings of the National Academy of Sciences of the United States of America, 105(36), 13592-13597.

McLean, A., Dowson, J., Toone, B., Young, S., Bazanis, E., Robbins, T. W., et al. (2004) Characteristic neurocognitive profile associated with adult attention-deficit/ hyperactivity disorder. Psychological Medicine, 34(4), 681-692.

Miller, E. K., \& Cohen, J. D. (2001). An integrative theory of prefrontal cortex function. Annual Review of Neuroscience, 24, 167-202.

Milner, B. (1963). Effects of different brain lesions on card sorting: The role of the frontal lobes. Archives of Neurology, 9(1), 90-100.

Neubert, F. X., Mars, R. B., Buch, E. R., Olivier, E., \& Rushworth, M. F. (2010). Cortical and subcortical interactions during action reprogramming and their related white matter pathways. Proceedings of the National Academy of Sciences of the United States of America, 107(30), 13240-13245.

Oberg, R. G., \& Divac, I. (1975). Dissociative effects of selective lesions in the caudate nucleus of cats and rats. Acta Neurobiologiae Experimentalis, 35(5-6), 647-659.

Owen, A. M., James, M., Leigh, P. N., Summers, B. A., Marsden, C. D., Quinn, N. P., et al. (1992). Fronto-striatal cognitive deficits at different stages of Parkinson's disease. Brain, 115, 1727-1751

Owen, A. M., Roberts, A. C., Hodges, J. R., \& Robbins, T. W. (1993). Contrasting mechanisms of impaired attentional set-shifting in patients with frontal lobe damage or Parkinson's disease. Brain, 116(5), 1159-1175.

Rogers, R. (1998). Dissociating executive mechanisms of task control following frontal lobe damage and Parkinson's disease. Brain, 121(5), 815-842.

Rogers, R. D., Andrews, T. C., Grasby, P. M., Brooks, D. J., \& Robbins, T. W. (2000). Contrasting cortical and subcortical activations produced by attentional-set shifting and reversal learning in humans. Journal of Cognitive Neuroscience, 12(1), 142-162.

Rougier, N. P., Noelle, D. C., Braver, T. S., Cohen, J. D., \& O’Reilly, R. C. (2005) Prefrontal cortex and flexible cognitive control: Rules without symbols. Proceedings of the National Academy of Sciences of the United States of America, 102(20), 7338-7343. 
van Schouwenburg, M. R., den Ouden, H. E. M., \& Cools, R. (2010). The human basal ganglia modulate frontal-posterior connectivity during attention shifting. Journal of Neuroscience, 30(29), 9910-9918.

van Schouwenburg, M. R., Zwiers, M. P., van der Schaaf, M. E., Geurts, D. E. M., Schellekens, A. F. A., Buitelaar, J. K., et al. (2013). Anatomical connection strength predicts dopaminergic drug effects on fronto-striatal function. Psychopharmacology, 227(3), 521-531.

Studholme, C., Constable, R. T., \& Duncan, J. S. (2000). Accurate alignment of functional EPI data to anatomical MRI using a physics-based distortion model. IEEE Transactions on Medical Imaging, 19(11), 1115-1127.

Taghzouti, K., Louilot, A., Herman, J. P., Le Moal, M., \& Simon, H. (1985). Alternation behavior, spatial discrimination, and reversal disturbances following 6-hydroxydopamine lesions in the nucleus accumbens of the rat. Behavioral and Neural Biology, 44(3), 354-363.

Tuch, D. S., Salat, D. H., Wisco, J. J., Zaleta, A. K., Hevelone, N. D., \& Rosas, H. D. (2005). Choice reaction time performance correlates with diffusion anisotropy in white matter pathways supporting visuospatial attention. Proceedings of the National Academy of Sciences of the United States of America, 102(34), $12212-12217$.

Visser, E., Qin, S., \& Zwiers, M. (2010). Echo-planar imaging distortion correction by constrained nonlinear coregistration improves group fMRI. In: Presented at the joint annual meeting ISMRM-ESMRMB, May 1-7, 2010, Stockholm, Sweden. poster \#3459.

Zwiers, M. P. (2010). Patching cardiac and head motion artefacts in diffusionweighted images. NeuroImage, 53(2), 565-575. 